\title{
BMJ Open Cohort profile: a data linkage cohort to examine health service profiles of people with intellectual disability in New South Wales, Australia
}

Simone Reppermund, ${ }^{1,2}$ Preeyaporn Srasuebkul, ${ }^{1}$ Theresa Heintze, ${ }^{1}$ Rebecca Reeve, ${ }^{1,3}$ Kimberlie Dean, ${ }^{4,5}$ Eric Emerson, ${ }^{6}$ David Coyne, ${ }^{7}$ Phillip Snoyman, ${ }^{8}$ Eileen Baldry, ${ }^{9}$ Leanne Dowse, ${ }^{10}$ Tracey Szanto, ${ }^{11}$ Grant Sara, ${ }^{12,13}$ Tony Florio, ${ }^{1}$ Julian N Trollor ${ }^{1,2}$

To cite: Reppermund $\mathrm{S}$, Srasuebkul P, Heintze T, et al. Cohort profile: a data linkage cohort to examine health service profiles of people with intellectual disability in New South Wales, Australia. BMJ Open 2017;7: e015627. doi:10.1136/ bmjopen-2016-015627

- Prepublication history and additional material is available. To view please visit the journal (http://dx.doi.org/ 10.1136/bmjopen-2016015627)

Received 20 December 2016 Revised 17 February 2017 Accepted 7 March 2017

CrossMark

For numbered affiliations see end of article.

Correspondence to Dr Simone Reppermund; s.reppermund@unsw.edu.au

\section{ABSTRACT}

Purpose: People with intellectual disability are a minority group who experience poorer physical and mental health than the general population and have difficulty accessing healthcare services. There is lack of knowledge about healthcare service needs and gaps experienced by people with intellectual disability. This study aims to interrogate a large linked administrative data set containing hospital admissions, presentations to emergency departments (ED) and mortality data to provide evidence to inform the development of improved health and mental health services for this population.

Participants: A retrospective cohort of people with intellectual disability ( $n=51$ 452) from New South Wales (NSW), Australia, to explore health and mental health profiles, mortality, pattern of health service use and associated costs between 2005 and 2013. The cohort is drawn from: the Disability Services Minimum Data Set; Admitted Patients Data Collection; Emergency Department Data Collection, Australian Bureau of Statistics Death Registry and Registry of Births, Deaths and Marriages. Mental health service usage among those with intellectual disability will be compared to a cohort of people who used mental health services $(n=1073139)$ and service usage other than for mental health will be compared with published data from the general population.

Findings to date: The median age of the cohort was 24 at the time of the last hospital admission and 21 at the last ED presentation. The cohort has a higher proportion of men than women and accounts for $0.6 \%$ of the NSW population in 2011 . Over $70 \%$ had up to 5 ED presentations and hospitalisations between 2005 and 2012. A high proportion of people with intellectual disability live in the most disadvantaged neighbourhoods.

Future plans: Results will be used to inform the development of more responsive healthcare, including improved interactions between health, social and disability supports. More generally, the results will assist the development of more inclusive policy frameworks for people with intellectual disability.

\section{Strengths and limitations of this study}

- This data linkage study builds a rich resource which allows the in-depth examination of the health profile and service contact of people with intellectual disability.

- In the Australian context of dispersed and relatively low population size, this is the most efficient methodology, yet in itself requires considerable time and financial resources.

- Results will be used to inform the development of more appropriate service models and policy frameworks for people with intellectual disability.

- Our study includes only people with intellectual disability who have received disability services for intellectual disability or who have been diagnosed or identified by the hospital or emergency department as having an intellectual disability. It does not contain primary healthcare records.

- Administrative data are not collected specifically for research but rather for record keeping and aggregate data purposes; therefore, some variables of potential interest are not available.

\section{INTRODUCTION}

People with intellectual disability are a minority group, accounting for $\sim 1 \%$ of the population. ${ }^{1}$ Also known as intellectual developmental disorder, intellectual disability, is an enduring condition involving impairment of general mental abilities which is first apparent during the developmental period and impacts significantly on the person's adaptive functioning. ${ }^{2}$ Typically, a person with intellectual disability has an extremely low IQ score (measured as two or more SDs below the population mean) with deficits in adaptive behaviours and a reduced capacity to engage in conceptual cognitive functions such as learning, reasoning and planning. ${ }^{2}$ 
Compared to the general population, people with intellectual disability are more likely to experience poor physical and mental health including complex health conditions such as epilepsy, sensory impairments, gastrointestinal problems, respiratory disorders, obesity, diabetes, osteoporosis and oral health problems. ${ }^{3}$ A lower life expectancy than the general population underscores the significant health inequality experienced by this population group. ${ }^{4}$

The prevalence of mental disorders is very high, with recent estimates in children and adolescents with intellectual disabilities indicating comorbidity rates of between $30 \%$ and $50 \% .^{5}$ Compared to the general population, people with intellectual disability experience higher rates of schizophrenia, affective disorders, anxiety disorders and dementia and rates of mental disorders in this population increase in keeping with the degree of disability. ${ }^{6-8}$ Schizophrenia has an earlier onset in people with intellectual disability, underscoring a specific developmental vulnerability to mental illness and the importance of timely access to psychiatric services for this group. ${ }^{9}$

Despite the over-representation of physical and mental disorders, access to health and mental health services for people with intellectual disability in Australia is limited and falls far short of that for the general population. ${ }^{11}{ }^{11}$ Barriers to effective health and mental healthcare for people with intellectual disability include: a lack of substantial epidemiological data on prevalence of physical and mental illness in people with intellectual disability; poor identification of people with intellectual disability due to masking and comorbidity; ${ }^{9}$ unavailability or lack of appropriate application of existing assessment instruments; discrimination in healthcare systems $;{ }^{12}$ a dearth of data on the interaction between, and distinct roles and responsibilities of, disability and mental health services; ${ }^{13}$ a lack of training and confidence of health professionals in treating people with intellectual disability; ${ }^{14-17}$ poor understanding by carers, ${ }^{18}$ disability and mental health workers of the manifestations of mental disorders in people with an intellectual disability; ${ }^{19}{ }^{20}$ a lack of coherent service models; inadequate funding for intellectual disability mental health services; ${ }^{13}$ poor coordination between services and treating agencies; ${ }^{21}$ scant services preventing involvement in the criminal justice system ${ }^{22}$ and a lack of specific inclusion of people with intellectual disability in the formulation of health and mental health policy.

Here we describe the creation of a linked administrative data set resource from which we explore the health and mental health profile and service use of people with intellectual disability. Potential analyses include descriptive profiling of the diagnoses given to people with intellectual disability within health service systems, characteristics and predictors of service use and costs and examination of health outcomes and their predictors. Comparisons can be made with the general population, as derived from the linked data set and publically available statistics. Although the main objective of the overall programme of work is to build a detailed profile of the health and health service system use of people with intellectual disability, the substantial unmet mental health needs of people with intellectual disability ${ }^{13}{ }^{23}$ and award of specific funding have created an imperative for a specific mental health subtheme.

The data linkage which forms the basis for this work has been made possible through a National Health and Medical Research Council Australia funded Partnerships for Better Health grant (ID: APP1056128; Title: Improving the Mental Health Outcomes of People with an Intellectual Disability), which is a larger collaborative project including academics, government and nongovernment organisations and people with intellectual disability. The broader partnership work has several themes including big data, qualitative work examining barriers and enablers to access and a national and state policy analysis. A cohesive knowledge translation framework has been developed which triangulates results from each theme and uses the findings to guide the development of healthcare services and policy for people with intellectual disability and mental illness at Australian Government and State Government level. The data linkage component has several benefits including large sample sizes and the potential for greater efficiency in time and resources of longitudinal data. Interrogation of linked data identifies the linkages and gaps between service sectors, and the benefit of cross-sector work.

\section{COHORT DESCRIPTION}

Administrative data sets relating to disability services, health services and mortality in NSW, Australia, have been linked at an individual level to allow an examination of the pattern and determinants of service use/ contact over time for those with established intellectual disability and those without known intellectual disability.

\section{Project resourcing and development}

Considerable time and resources have been required to develop the data linkage component of the study. Piloting of the project occurred in 2012, when the team sought approval to link a subset of one-third of the NSW ambulatory mental health data set to the Disability Services Minimum Data set (DS-MDS). Following proof of concept and publication of initial results ${ }^{24}$ further funding was obtained to undertake the current linkage. Overall resourcing to date has been substantial and includes approximately 2 years full-time salary equivalents for a research officer, 2-year full-time salary equivalent for a data analyst, oversight of the project by a senior academic, substantial costs associated with linkage and storage of data sets and multiple in kind contributions from partner agencies and collaborators.

\section{Overview of data sources}

There is no single registry which collects information about people with intellectual disability. In this project, 
multiple data sets have been used to help identify those with intellectual disability. All people identified as having intellectual disability fulfilled either Diagnostic and Statistical Manual of Mental Disorders (DSM) IV or International Statistical Classification of Diseases and Related Health Problems 10th revision (ICD-10) criteria for a diagnosis of intellectual disability.

\section{Disability services data}

The DS-MDS is a de-identified data set which collates information about people receiving disability services in NSW, including the nature of their disability and the services provided to persons with a disability. The main services provided by the agency, Ageing, Disability and Home Care (ADHC), include accommodation, community support, community access and respite. Such information is routinely collected by each Australian State and Territory under the National Disability Agreement. ${ }^{25}$ Given the services provided, the DS-MDS contains information on service recipients' demographics, living arrangements, support needs, carers and services received. A full list of the variables in this data set is presented in online supplementary file 1 . The DS-MDS includes $\mathrm{n}=73674$ children and adults who resided in NSW and who were registered to receive a disability service between 01 July 2005 and 30 June 2012. From this data set, a total of 42243 people with intellectual disability was identified. Fulfilment of DSM IV criteria for intellectual disability was required in order to be eligible to receive a service due to intellectual disability.

\section{Health services data}

\section{NSW Admitted Patient Data Collection}

The NSW Admitted Patient Data Collection (APDC) is a de-identified data set which collates information on all admitted patient services provided by NSW public hospitals, public psychiatric hospitals, public multipurpose services, private hospitals and private day procedures centres. It contains dates of admissions and separations for each episode of care, up to 50 diagnoses relevant to each episode of care, the source of referral, separation mode and procedures based on ICD-10 Australian version. ${ }^{26}$ The current project includes APDC data on separations that occurred between 1 January 2005 and 30 June 2012 for 1016446 people. A list of the variables in this data set is included in online supplementary file 2.

\section{NSW Emergency Department Data Collection}

The NSW Emergency Department Data Collection (EDDC) is a de-identified data set which collates information on presentations to an emergency department (ED) in a NSW public hospital. It includes dates and times of presentation and discharge, reason for presentation, triage category and outcome of the presentation (discharge, transfer or death). The study includes data from 1 January 2005 to 30 June 2012 for 188359 people. There are $150 \mathrm{ED}$ centres in NSW, and 90 $(60 \%)$ of those participated in the data collection. ${ }^{27}$ Although only $60 \%$ of the ED centres participated in the data collection, these reporting centres are larger centres hence a substantial proportion of the presenting population is covered. A listing of variables in the EDDC is displayed in online supplementary file 3 .

\section{Mortality data}

There are two data sets containing mortality information. Observations in both data sets are based on the year of registration of the death rather than the year the death occurred, although in most cases these are equivalent. The mortality information available for linkage includes only people who died in NSW.

\section{NSW Registry of Births, Deaths and Marriages Data}

The NSW Registry of Births, Deaths and Marriages (RBDM) registers all deaths that occur in NSW and contains raw and uncoded data in the death certificate. Data are available from January 2005 to June 2013. A listing of variables in the RBDM is displayed in online supplementary file 4 .

\section{Australian Bureau of Statistics Deaths Registration Data}

The Australian Bureau of Statistics (ABS) Deaths Registration Data contains ICD-10 international version coded causes of death information and date of death. For our project, ICD-10 coded causes of deaths were available until 2007. A list of variables is included in online supplementary file 4. Data are available from January 2005 to December 2007.

\section{Cohort definition}

Our data linkage contains the records of a cohort of people with intellectual disability who have ever received disability services in NSW recorded in the DS-MDS described above $(n=73674)$ and those who have been identified as having intellectual disability through diagnosis codes in the APDC and the EDDC in a NSW hospital. These 'intellectual disability' codes are: F700-F701; F708-F709; F710-F711; F718-F719; F720-F721; F728-F729; F730-F731; F728-F729; F730- F731; F738-F739; F780-F781; F788-F791; F843-F844; F798-F799; Q900-Q902; Q909; Q910-Q912; Q913; Q914-Q916; Q917; Q930-Q939; Q992; P043; Q860; Q861; Q862; Q868 Q870-Q873; Q875; Q878; Q898. These codes include intellectual disability due to childhood disintegrative and overactive disorders associated with mental retardation, intellectual development delay, mild through profound mental retardation, Down syndrome and other chromosomal anomalies associated with mental retardation, Fragile X syndrome and congenital malformation syndromes due to known exogenous causes. Those diagnosed with autism without a co-occurring intellectual disability are not defined as having an intellectual disability for the purposes of this project. The intellectual disability cohort accounts for 
$0.6 \%$ of the NSW population in 2011, and people with mild intellectual disability may be underrepresented.

To compare mental health profile and service usage in people with and without intellectual disability, a cohort comprising people who either used mental health services or who had a mental health diagnosis, regardless of the intellectual disability status, was also formed ( $n=1073$ 139). People in this cohort had at least one admission to a psychiatric ward or were diagnosed with at least one mental health ICD10 code (F00-F25, F28-F48, F50, F51, F53-F73, F78-F91, F93-F99) during any hospital admission (psychiatric or non-psychiatric) or during any presentation to an ED. We then link these data to the intellectual disability status in order to quantify and compare rates and patterns of mental ill-health in people with intellectual disability and the general population.

For other health usage, we will compare the results of our cohort with information published by HealthStats NSW or the Australian Institute for Health and Welfare (AIHW). We will calculate rates of ED presentations, non-mental health hospital episodes and death in our cohort using appropriate data sets. For comparison with the general population, we will use AIHW published results for ED presentations in NSW and all cause hospitalisations in NSW, as well as death from HealthStats NSW. To make direct comparisons between the cohort of people with intellectual disability and the general population, we will standardise the usage rates on age and sex using the Australian Standard Population. If published data permit, we will adjust for the impact of deprivation using the regression method.

\section{Data linkage}

Linkage of the aforementioned data sets was performed by the NSW Centre for Health Record Linkage (CHeReL). The CHeReL maintains a linkage system for health-related data in NSW in accordance with all ethical, legal, privacy and confidentiality requirements. The CHeReL keeps a Master Linkage Key (MLK) that consists of continuously updated links between most NSW Health data sets. The CheReL does not house the data; all data sets are kept by the respective data custodians.

Individuals in the DS-MDS were identified and their records matched in a deterministic manner using a Statistical Linkage Key (SLK581) identifier. Records in the APDC, EDDC and RBDM are matched to individuals using an MLK. ${ }^{28}$ The CHeReL created an SLK581 identifier for the matched APDC, EDDC and RBDM records and linked this with the SLK581 keys in the DS-MDS. We do not have information on the false-positive rate using the deterministic approach; however, it is expected to be higher than 5/1000 aimed for by the CHeReL. Currently, the CHeReL linked 43772 (59\%) records of people in DS-MDS to APDC, EDDC, ABS or RBDM and $29902(41 \%)$ records did not link to APDC, EDDC, ABS or RBDM.
Data custodians provided the CHeReL with an encrypted client number and relevant personal information for all clients over the relevant time periods. The CHeReL linked the DS-MDS database to the NSW data collections of APDC, EDDC, ABS and RBDM using the linkage method described above and provided each data custodian a project person number (PPN) and an encrypted client number for each database. The data custodians decrypted the source record number and merged the PPN with their data sets for use in this project. The source record number was removed, and the researchers were provided with de-identified files containing only the PPN and relevant study variables. The PPN allowed for merging the various data sets as needed.

\section{Data cleaning and plan of analyses}

Once the linked data were received, a data cleaning process was carried out including checking for unexpected trends, checking that the data were complete with all requested variables available and a validity check. People who appeared with a different sex or different data of birth/date of death in different data sets were excluded from the data set.

The analyses described in this paper include the demographic profiles such as age, sex, area of residence and socioeconomic status, as well as health resource usage for people with intellectual disability.

\section{Ethics}

Ethics approval was obtained from the NSW Population and Health Services Research Ethics Committee (AU RED Study Reference Number: HREC/13/CIPHS/7; CINSW Reference Number: 2013/02/446), and access to the data sets was granted by relevant data custodians. An ethics requirement is that the linked data can only be analysed at the physical location of the researchers undertaking this work (The Department of Developmental Disability Neuropsychiatry, UNSW Sydney). The timeframe from submitting the ethics application to ethics approval was $\sim 12$ months and another 8 months from ethics approval to receipt of the data.

\section{FINDINGS TO DATE}

Figure 1 shows the number of people identified as having intellectual disability in the DS-MDS, APDC and EDDC data sets. Percentages of people with intellectual disability in each individual data set are $82 \% \quad(n=42243)$ in the DS-MDS; $47 \%(\mathrm{n}=24242)$ in the EDDC and $55 \%$ $(n=28233)$ in the APDC. A total of $34 \% \quad(n=17267)$ appear in all three data sets, 6\% ( $\mathrm{n}=2932)$ appear only in the DS-MDS and the APDC, $10 \%(n=5037)$ appear in the DS-MDS and the EDDC and 4\% ( $\mathrm{n}=1898)$ appear in the EDDC and the APDC. Note that in order to be included in our cohort, an individual had to receive a service with an intellectual disability flag. However, not all people with intellectual disability would necessarily 


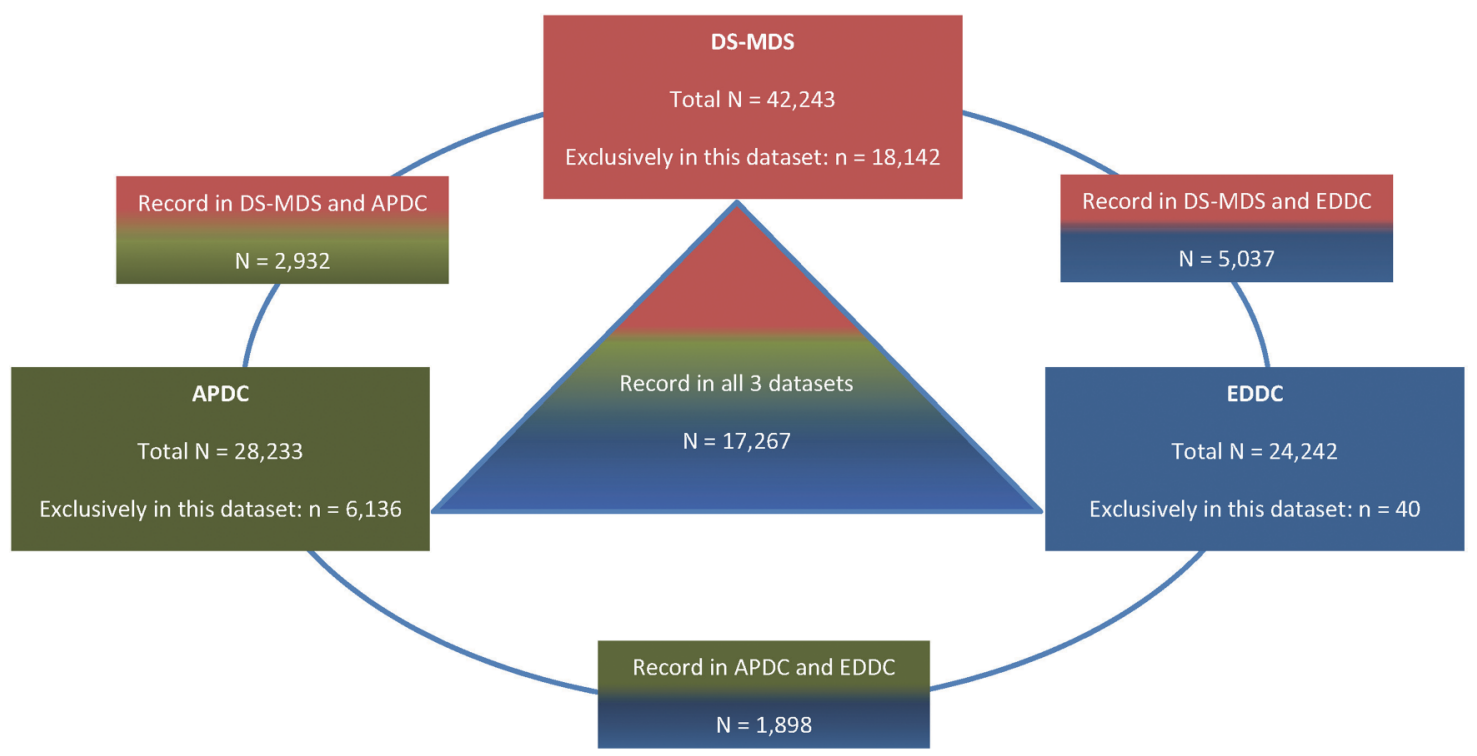

Note: the total cohort of people with intellectual disability is $n=51,452$, comprising of $n=18,142$ from the DS-MDS, 6,136 from the APDC, $n=40$ from the EDDC, $n=2,932$ with simultaneous records in the DS-MDS \& APDC, $n=5,037$ with simultaneous records in the DS-MDS and EDDC, $n=1,898$ with simultaneous records in the APDC and EDDC and $n=17,267$ with records in all 3 datasets.

Figure 1 Number of people identified as having intellectual disability in the DS-MDS, APDC and EDDC data sets. APDC, Admitted Patient Data Collection; DS-MDS, Disability Services Minimum Data set; EDDC, Emergency Department Data Collection.

have a hospital admission or ED presentation record. Overall, $82 \%$ of the cohort received disability services due to their intellectual disability. Of the remaining $18 \%$ who did not receive disability services due to intellectual disability, $2.3 \%$ received disability services for nonintellectual disability related needs.

Table 1 displays the demographics of the study population in each data set DS-MDS, APDC, EDDC and RBDM. The data shown is on a person level, and a person may have multiple records in the full analysis period of 20052012. Our cohort comprises 51452 people with intellectual disability with a median age of 24 at the time of their last admission to a hospital or day procedure centre and a median age of 21 at their last presentation to an ED. The median age of death is 56 years. The cohort has a higher proportion of men than women: across the data sets, the proportions of men range between $57 \%$ and $60 \%$. Two-thirds of people live in a major city and about one-quarter lives in an inner regional city and $6 \%$ live in outer regional cities. Across all health services, only $15 \%$ of people with intellectual disability live in the least disadvantaged neighbourhoods.

Table 2 presents information from the APDC and EDDC data sets on a record level (ie, multiple records for one person). As the RBDM is the same whether it is presented at a person (table 1) or record level, it has not been retabulated in table 2 .

Proportions of intellectual disability are similar on a record level and on a person level basis. Percentages of the records in the APDC and in EDDC that were defined as from people with intellectual disability are $3 \%$ and $12 \%$, respectively. Consistent across all data sets a higher proportion of men with intellectual disability receive health services than women (tables 1 and 2).

The proportion of people who received disability services is lower when assessed at the record level $(59 \%$ and $78 \%$ ) than on the person level $(72 \%$ and $92 \%)$, in the APDC and EDDC data sets. This indicates that those who have ever received disability services have, on average, fewer ED presentations and admissions to hospital than those who have not received disability services.

Overall, for the APDC and EDDC, there are on average eight records per person in the full record database. The number of hospitalisations (data from the APDC) is displayed in figure 2, and the number of ED presentations (data from the EDDC) is displayed in figure 3. The distribution of the number of hospitalisations (APDC) and ED presentations (EDDC) is highly skewed.

As seen in figures 2 and 3, over $70 \%$ of people with intellectual disability have up to five ED presentations and hospitalisations.

\section{Future directions}

Four major themes will be the focus of the project: hospital admissions, ED presentations, mortality and costs. Analyses will include predictors of hospital admission and re-admission, frequency and length of stay for health and mental health admissions. We will investigate the demographics of people with intellectual disability 
Table 1 Health and intellectual disability service and mortality profiles as recorded in the data set person level (at the last record), numbers presented in $\mathrm{n}(\%)$ unless otherwise specified

\begin{tabular}{|c|c|c|c|c|}
\hline & APDC & EDDC & $\begin{array}{l}\text { Mortality } \\
\text { (RBDM) }\end{array}$ & DS-MDS \\
\hline $\begin{array}{l}\text { Number of people (\% of the defined cohort with } \\
\text { intellectual disability) }\end{array}$ & $28233(55)$ & $24242(47)$ & $2844(5.5)$ & $42243(82)$ \\
\hline Median (IQR) age at the last event, years & $24(10-46)$ & $21(10-41)$ & $56(35-68)$ & $23(14-42)$ \\
\hline $0-4$ & $3408(12.1)$ & $2115(8.7)$ & $160(5.6)$ & $1022(2.4)$ \\
\hline $5-14$ & $5878(20.8)$ & $6197(25.6)$ & $149(5.2)$ & $10258(24.3)$ \\
\hline $15-24$ & $4955(17.6)$ & $5202(21.5)$ & $194(6.8)$ & $10987(26.0)$ \\
\hline $25-34$ & $3279(11.6)$ & $3048(12.6)$ & $181(6.4)$ & $5842(13.8)$ \\
\hline $35-44$ & $3210(11.4)$ & $2618(10.8)$ & $246(8.7)$ & $4832(11.4)$ \\
\hline $45-54$ & $2993(10.6)$ & $2361(9.7)$ & $424(14.9)$ & $4511(10.7)$ \\
\hline $55-64$ & $2466(8.7)$ & $1768(7.3)$ & $556(19.6)$ & $3237(7.7)$ \\
\hline $65-74$ & $1306(4.6)$ & $688(2.8)$ & 494 (17.4) & $1203(2.9)$ \\
\hline $75-84$ & $580(2.1)$ & $203(0.8)$ & $323(11.4)$ & $284(0.7)$ \\
\hline 85 and over & $158(0.6)$ & $42(0.2)$ & $117(4.1)$ & $67(0.2)$ \\
\hline \multicolumn{5}{|l|}{ Sex } \\
\hline Female & $11753(41.6)$ & 9529 (39.3) & $1214(42.7)$ & $16885(40.0)$ \\
\hline Male & $16480(58.4)$ & $14712(60.7)$ & 1629 (57.3) & $25334(60.0)$ \\
\hline Other & 0 & $1(0.0)$ & $1(0.0)$ & $24(0.1)$ \\
\hline Ever received disability services & 20199 (71.5) & 22304 (92.0) & $1448(50.9)$ & $42243(100)$ \\
\hline \multicolumn{5}{|l|}{ Remoteness area of residence } \\
\hline Major cities & $19042(67.5)$ & $16215(66.9)$ & $1993(70.1)$ & $28137(66.6)$ \\
\hline Inner regional & 7017 (24.9) & 6524 (26.9) & $639(22.5)$ & $11254(26.6)$ \\
\hline Outer regional & $1791(6.3)$ & $1295(5.3)$ & $183(6.4)$ & $2545(6.0)$ \\
\hline Remote & $137(0.5)$ & $85(0.4)$ & $9(0.3)$ & $197(0.5)$ \\
\hline Very remote & $7(0.0)$ & $8(0.0)$ & $1(0.0)$ & $9(0.0)$ \\
\hline Unknown & $239(0.9)$ & $115(0.5)$ & $19(0.7)$ & $0(0.0)$ \\
\hline \multicolumn{5}{|c|}{ Index of relative socioeconomic disadvantage in NSW of residence } \\
\hline First quintile (most disadvantaged) & $5633(20.0)$ & $4864(20.1)$ & $533(18.7)$ & $5803(13.7)$ \\
\hline Second quintile & $5563(19.7)$ & $4853(20.0)$ & $588(20.7)$ & $6125(14.5)$ \\
\hline Third quintile & $7314(25.9)$ & $6569(27.1)$ & $736(25.9)$ & $8258(19.6)$ \\
\hline Fourth quintile & $5523(19.6)$ & 4655 (19.2) & $560(19.7)$ & $5902(14.0)$ \\
\hline Fifth quintile (least disadvantaged) & $3965(14.0)$ & 3190 (13.2) & $408(14.4)$ & 4439 (10.5) \\
\hline Unknown & $235(0.8)$ & $111(0.5)$ & $19(0.7)$ & $11716(27.7)$ \\
\hline
\end{tabular}

who present at the ED, their rate of service use, arrival mode and whether ED presentations were considered a GP-type presentation. Mortality rates and predictors of mortality in people with intellectual disability will be examined as well as associations between use of disability services and comorbidities on mortality. Finally, costs of hospital services will be investigated.

\section{STRENGTHS AND LIMITATIONS}

Our linkage approach enables the inclusion of a broad range of people with intellectual disability in NSW, the most populous state in Australia. Similarities in demography between most states and territories in Australia enhance the generalisability of our results to Australian service users with an intellectual disability.

The data linkage enables us to conduct analyses examining patterns of service use related to different components of the health service system (inpatient, emergency, adult services, children and younger people's services), and costs associated with healthcare, and mortality, cause and predictors of death. A greater understanding of service and indices of health system efficiency for people with intellectual disability will emerge, for example, through the frequency and timeframe of readmissions to hospital, representations to ED and their predictors. The inclusion of an additional mental health cohort in our data set will allow a direct comparison between the mental health profile and service use of people with and without intellectual disability.

The analysis of linked health and disability service data fills a current gap in the Australian knowledge base regarding the health profile and service system needs of intellectual disability. These data will be triangulated with the other two main projects within this programme of research, to improve access to and quality of healthcare for people with intellectual disability. 
Table 2 Health service profiles as recorded in the data set (record level), numbers presented in $n(\%)$ unless otherwise specified

\begin{tabular}{|c|c|c|}
\hline & APDC & EDDC \\
\hline Total records & 225904 & 200868 \\
\hline Date ranges & 1 January 2005-30 June 2012 & 1 January 2005-30 June 2012 \\
\hline Median (IQR) age at the event, years & $29(11-49)$ & $26(13-42)$ \\
\hline $0-4$ & $31889(14.1)$ & $23545(11.7)$ \\
\hline $5-14$ & $35322(15.6)$ & 30448 (15.2) \\
\hline $15-24$ & 32967 (14.6) & $40385(20.1)$ \\
\hline $25-34$ & $25602(11.3)$ & 32820 (16.3) \\
\hline $35-44$ & 30241 (13.4) & 31910 (15.9) \\
\hline $45-54$ & $29269(13.0)$ & $22823(11.4)$ \\
\hline $55-64$ & $20473(9.1)$ & $13578(6.8)$ \\
\hline $65-74$ & $11991(5.3)$ & $4041(2.0)$ \\
\hline $75-84$ & $711(3.2)$ & $1100(0.6)$ \\
\hline 85 and over & $1038(0.5)$ & $213(90.1)$ \\
\hline Invalid data & $1(0.0)$ & $5(0.0)$ \\
\hline \multicolumn{3}{|l|}{ Sex } \\
\hline Female & $100387(44.4)$ & $87930(43.8)$ \\
\hline Male & $125515(55.6)$ & 112927 (56.2) \\
\hline Other/missing & $2(0.0)$ & $11(0.0)$ \\
\hline Ever received disability services & $133437(59.1)$ & $156038(77.7)$ \\
\hline \multicolumn{3}{|l|}{ Remoteness area of residence } \\
\hline Major cities & $161762(71.9)$ & $133770(66.6)$ \\
\hline Inner regional & $45433(21.1)$ & $52436(26.1)$ \\
\hline Outer regional & $13897(5.8)$ & $11343(5.7)$ \\
\hline Remote & $2147(0.6)$ & $372(0.2)$ \\
\hline Very remote & $61(0.0)$ & $38(0.0)$ \\
\hline Unknown & $2604(0.7)$ & $2909(1.5)$ \\
\hline \multicolumn{3}{|c|}{ Index of relative socioeconomic disadvantage in NSW of residence } \\
\hline First quintile (most disadvantaged) & $47338(21.0)$ & 44672 (22.2) \\
\hline Second quintile & $42141(18.7)$ & 40735 (20.3) \\
\hline Third quintile & $55231(24.5)$ & $53752(26.8)$ \\
\hline Fourth quintile & $45153(20.0)$ & 38005 (18.9) \\
\hline Fifth quintile (least disadvantaged) & $33455(14.8)$ & $20818(10.4)$ \\
\hline Unknown & $2586(1.4)$ & $2886(1.4)$ \\
\hline
\end{tabular}

Our results will inform sector and services development. In light of the Australian rollout of the National Disability Insurance Scheme (NDIS) including in NSW, the project is an important source for informing policy and practices to improve the coordination between health and disability sectors. Our study will establish baseline health and mental health profiles, service use and costs across multiple components of the health services system, against which NDIS-related change can be interrogated.

A number of challenges have been encountered in establishing this data infrastructure. The data linkage process is time-intensive and resource-intensive. Researchers need to be aware that the process of applying for, combining and cleaning these data sets can take months or even years and requires experienced data analysts. In particular the relatively lengthy process of applying for/waiting for the linked data and receiving ethics clearance can be a major challenge for research projects that are only funded from external sources for a limited amount of time. As with most administrative data sets, data have been collected for administrative rather than clinical purposes, and as such has significant shortcomings. For example, in the hospitalisation data, if a person had multiple diagnoses in one episode, we do not have information on the length of each diagnosis or the severity of it. Coverage is limited in three respects. Our data do not reflect all ED presentations because not all departments contribute to this minimum data set. Importantly however, the majority of people live in areas with contributing EDs, so the impact is minimal in our study. We cannot identify all people with intellectual disability, rather those who have received disability services for intellectual disability or who have been diagnosed or identified by the hospital or ED as having an intellectual disability. Therefore, we are missing those individuals with intellectual disability who were not considered eligible for disability services and in whom intellectual disability was not recognised or coded by health services. The excluded individuals are highly unlikely to be a random subset of those with intellectual disability; rather they are more likely to be people with milder 


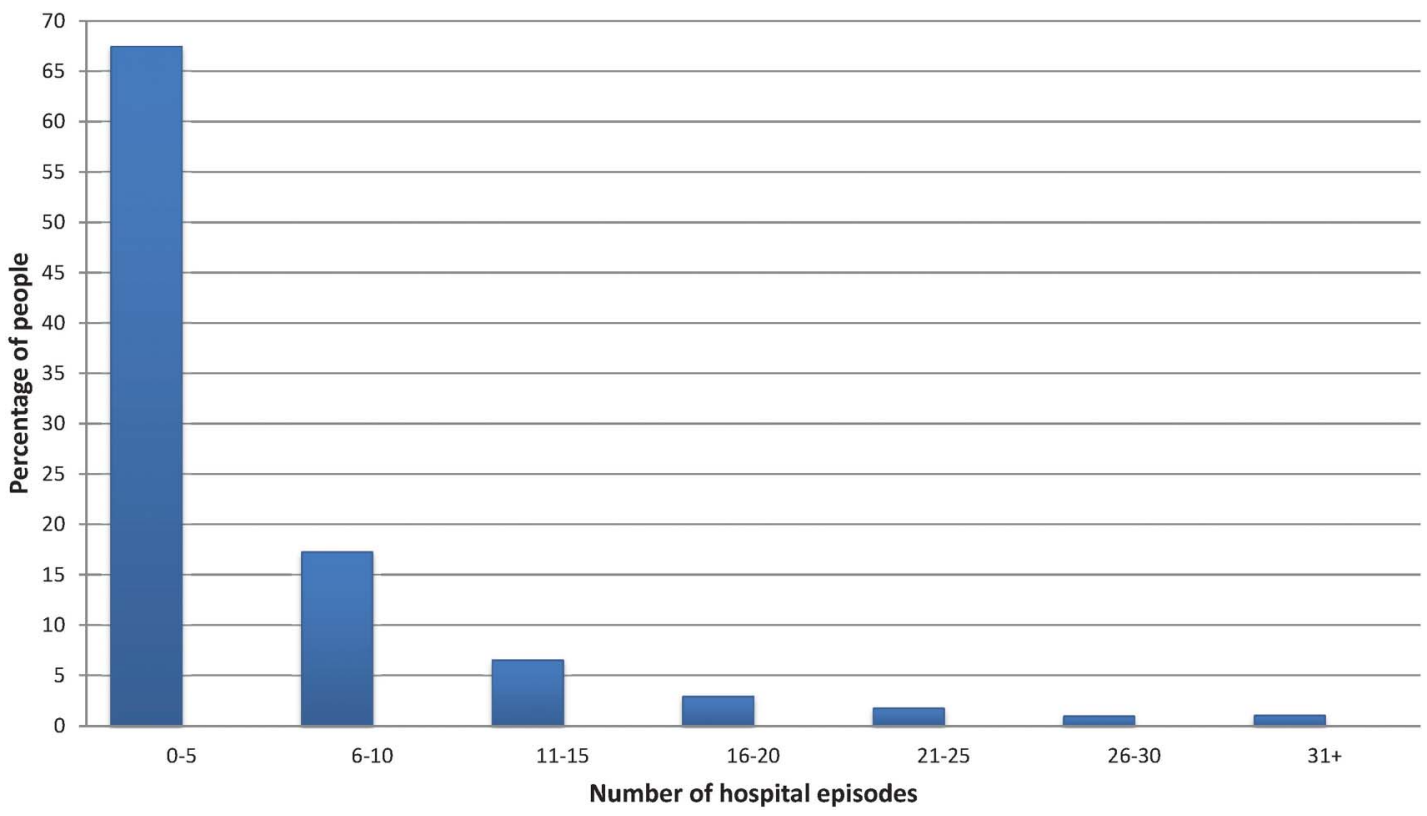

Figure 2 Number of hospital admission for people with intellectual disability between January 2005 and June 2012.

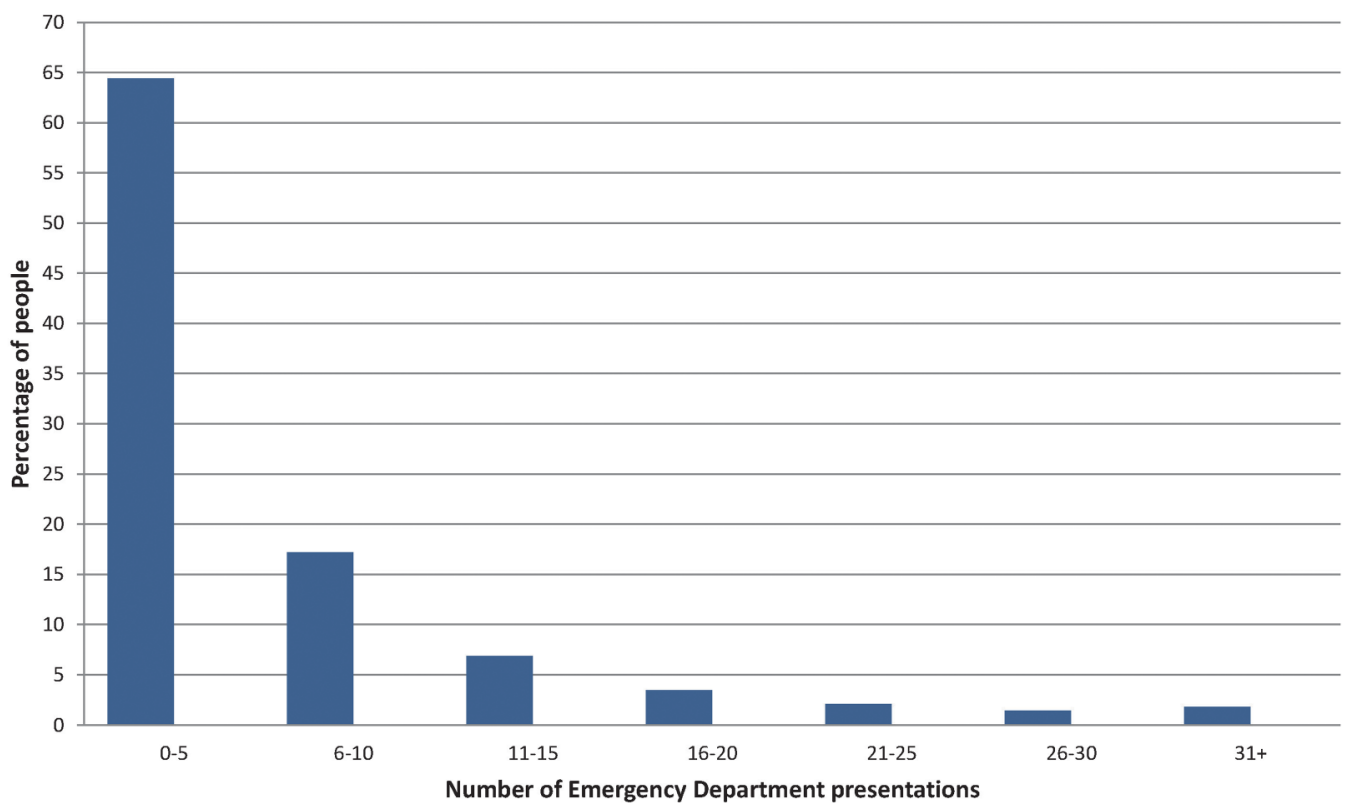

Figure 3 Number of ED presentations for people with intellectual disability between January 2005 and June 2012 . ED, Emergency Department.

intellectual disability and/or with additional disadvantage which limits access to services. Additional linkage to other data sets with intellectual disability identifiers would overcome this problem to some extent. Further, with the exception of the ability for direct comparison within mental health services, we do not have person level data of the general NSW population and hence need to compare other data-points to publically available data sources.

Record linkage can sometimes erroneously make falsepositive links or fail to link when a true link exists (false negative). Additionally, administrative data are not collected specifically for research but rather for record keeping and aggregate data purposes. Some variables, for example, relating to severity of disability or measures of adaptive behaviour, that we would like to include in our models are not available in the data. In turn, this may increase the chances of omitted variable bias in our models. The current linkage does not include community health services or general practitioner records which may add additional value to the analyses as it requires linking data from different jurisdiction and not 
feasible at the time of this study. Finally, our cohort with intellectual disability is heterogeneous as we used multiple data sources with differences in definition or context of diagnosis of intellectual disability, which can be easily adjusted in the analyses.

An update of the cohort with inclusion of additional data is currently in progress. Specifically, we will add data from Corrective Services NSW, NSW Department of Education and NSW Public Guardian and we will extend the timeframe to 2001-2016. This will allow us to identify, quantify and cost health and other services provision to people with ID within the various cohorts of interest.

In conclusion by interrogating the linked disability and health data sets and triangulating this with data derived from an analysis of Commonwealth and State Mental Health Policy and a qualitative research approach with stakeholder engagement to improve accessibility, this project will inform the development of more appropriate service models and policy frameworks for people with intellectual disability.

\section{COLLABORATION}

Initial data analyses and publications will be generated by investigators on the NHMRC partnerships for better health: improving the mental health outcomes for people with an intellectual disability. However, the research team is open to potential research collaborations; researchers interested in collaboration should contact the corresponding author with their expression of interest. Access to the data and analytical files is only permitted with the expressed permission of the approving human research ethics committees and data custodians. Analysis of linked data is currently authorised to occur at only one location, owing to ethical considerations.

\section{Author affiliations}

${ }^{1}$ Department of Developmental Disability Neuropsychiatry, School of Psychiatry, UNSW Australia, Sydney, New South Wales, Australia

${ }^{2}$ Centre for Healthy Brain Ageing, School of Psychiatry, UNSW Australia, Sydney, New South Wales, Australia

${ }^{3}$ Centre for Social Impact, Sydney, New South Wales, Australia

${ }^{4}$ School of Psychiatry, UNSW Australia, Sydney, New South Wales, Australia

${ }^{5}$ Justice Health \& Forensic Mental Health Network, Sydney, New South Wales, Australia

${ }^{6}$ Centre for Disability Research and Policy, University of Sydney, Sydney, New South Wales, Australia

${ }^{7}$ Ageing, Disability and Home Care, Department of Family and Community Services NSW, Sydney, New South Wales, Australia

${ }^{8}$ Corrective Services NSW, Department of Justice, Sydney, New South Wales, Australia

${ }^{9}$ Faculty of Arts and Social Sciences, School of Social Sciences, UNSW Australia, Sydney, New South Wales, Australia

${ }^{10}$ Intellectual Disability Behaviour Support Program, School of Social

Sciences, UNSW Australia, Sydney, New South Wales, Australia

${ }^{11}$ Intellectual Disability Network, NSW Agency for Clinical Innovation, Sydney, New South Wales, Australia

${ }^{12}$ InforMH, Health System Information and Performance Reporting Branch, NSW Ministry of Health, Sydney, New South Wales, Australia

${ }^{13}$ School of Psychiatry, Sydney Medical School, University of Sydney, Sydney, New South Wales, Australia
Twitter Follow Department of Developmental Disability Neuropsychiatry @3DN_UNSW

Acknowledgements The authors acknowledge all investigators and partner organisations contributing to the project: Cls: Julian Trollor, Eric Emerson, Rhoshel Lenroot, Karen Fisher, Kimberlie Dean, Leanne Dowse.

Als: Eileen Baldry, Tony Florio, Grant Sara, Phillip Snoyman, Les White.

Project staff and students: Angela Dew, Preeyaporn Srasuebkul, Erin Whittle, Simone Reppermund, Snow Lee, Bronwyn Newman, Theresa Heintze.

Partner organisations: Agency for Clinical Innovation-Intellectual Disability Network, NSW Department of Family \& Community Services-Ageing, Disability and Home Care, NSW Department of Education, NSW Department of Justice-Corrective Services NSW, NSW Ministry of Health-Justice Health \& Forensic Mental Health Network, Mental Health Commission of NSW, NSW Ministry of Health-Mental Health \& Drug \& Alcohol Office, NSW Ministry of Health-InforMH, Mental Health Review Tribunal, National and NSW Council for Intellectual Disability, National Disability Services, NSW Office of the Public Guardian, NSW Ombudsman.

Contributors JNT conceived and designed the study. KD, EE, DC, PS, EB, LD, TS, GS and TF participated in the conceptual design of the study. SR, PS, TH and JNT drafted the manuscript. PS and TH performed the analyses. TF provided statistical support. RR provided expertise about health economics. All authors critically revised the manuscript and contributed to interpretation of the data. All authors read and approved the final version of the manuscript.

Funding The study is part of a National Health and Medical Research Council Australia funded Partnerships for Better Health grant (ID: APP1056128; Title: Improving the Mental Health Outcomes of People with an Intellectual Disability).

Competing interests None declared.

Patient consent No.

Ethics approval Ethics approval was obtained from the NSW Population and Health Services Research Ethics Committee (AU RED Study Reference Number: HREC/13/CIPHS/7; CINSW Reference Number: 2013/02/446), and access to the data sets was granted by relevant data custodians.

Provenance and peer review Not commissioned; externally peer reviewed.

Data sharing statement Direct access to the data and analytical files is not permitted without the expressed permission of the approving human research ethics committees and data custodians. Researchers interested in collaboration should contact the corresponding author with their expression of interest.

Open Access This is an Open Access article distributed in accordance with the Creative Commons Attribution Non Commercial (CC BY-NC 4.0) license, which permits others to distribute, remix, adapt, build upon this work noncommercially, and license their derivative works on different terms, provided the original work is properly cited and the use is non-commercial. See: http:// creativecommons.org/licenses/by-nc/4.0/

\section{REFERENCES}

1. Maulik PK, Mascarenhas MN, Mathers CD, et al. Prevalence of intellectual disability: a meta-analysis of population-based studies. Res Dev Disabil 2011;32:419-36.

2. American Psychiatric Association. Diagnostic and statistical manual of mental disorders. 5th edn. Washington (DC): APA, 2013.

3. Hatton C, Emerson E. Introduction: health disparities, health inequity, and people with intellectual disabilities. In: Hatton C, Emerson E. eds. Health disparities and people with intellectual disabilities. International review of research in developmental disabilities. New York: Elsevier, 2015.

4. Bittles $A H$, Petterson BA, Sullivan SG, et al. The influence of intellectual disability on life expectancy. J Gerontol A Biol Sci Med Sci 2002;57:M470-2.

5. Einfeld SL, Ellis LA, Emerson E. Comorbidity of intellectual disability and mental disorder in children and adolescents: a systematic review. J Intellect Dev Disabil 2011;36:137-43.

6. Cooper SA, Holland A. Dementia and mental ill-health in older people with intellectual disabilities. In: Bouras N, Holt G, eds. $P$ sychiatric and behavioural disorders in intellectual and 
developmental disabilities. Cambridge, UK: Cambridge University Press, 2007.

7. Strydom A, Livingston G, King M, et al. Prevalence of dementia in intellectual disability using different diagnostic criteria. $\mathrm{Br}$ J Psychiatry 2007;191:150-7.

8. Borthwick-Duffy SA. Epidemiology and prevalence of psychopathology in people with mental retardation. J Consult Clin Psychol 1994;62:17-27.

9. Morgan VA, Leonard $\mathrm{H}$, Bourke $\mathrm{J}$, et al. Intellectual disability co-occurring with schizophrenia and other psychiatric illness: population-based study. Br J Psychiatry 2008;193:364-72.

10. Evans E, Howlett S, Kremser T, et al. Service development for intellectual disability mental health: a human rights approach. J Intellect Disabil Res 2012;56:1098-109.

11. Emerson E. Hatton C. Health inequalities and people with intellectual disabilities. Cambridge: Cambridge University Press, 2014.

12. Llewellyn G, Vaughan C, Emerson E. Discrimination and the health of people with intellectual disabilities. In: Hatton C, Emerson E. eds. Health disparities and people with intellectual disabilities. International review of research in developmental disabilities. New York: Elsevier, 2015.

13. Evans E, Howlett S, Kremser T, et al. Service development for intellectual disability mental health: a human rights approach. $J$ Intellect Disabil Res 2012;56:1098-109.

14. Edwards N, Lennox N, White P. Queensland psychiatrists' attitudes and perceptions of adults with intellectual disability. J Intellect Disabil Res 2007;51(Pt 1):75-81.

15. Jess G, Torr J, Cooper S-A, et al. Specialist versus generic models of psychiatry training and service provision for people with Intellectual disabilities. J Appl Res Intellect Disabil 2008;21:183-93.

16. Phillips A, Morrison J, Davis RW. General practitioners' educational needs in intellectual disability health. J Intellect Disabil Res 2004;48 (Pt 2):142-9.

17. Torr J, Lennox N, Cooper SA, et al. Psychiatric care of adults with intellectual disabilities: changing perceptions over a decade. Aust $N$ Z J Psychiatry 2008;42:890-7.

18. Moss S, Emerson E, Bouras N, et al. Mental disorders and problematic behaviours in people with intellectual disability: future directions for research. J Intellect Disabil Res 1997:41:440-7.

19. Costello $\mathrm{H}$, Bouras $\mathrm{N}$, Davis $\mathrm{H}$. The role of training in improving community care staff awareness of mental health problems in people with intellectual disabilities. J Appl Res Intellect Disabil 2007;20:228-35.

20. Patel P, Goldberg D, Moss S. Psychiatric morbidity in older people with moderate and severe learning disability. II: the prevalence study. Br J Psychiatry 1993;163:481-91.

21. Cohen K. Mental health table forum. Which doors lead to where? How to enhance access to mental health service: barriers, facilitators and opportunities for Canadians' Mental Health. Ottawa: Canadian Psychological Association, 2010.

22. Baldry E, Clarence M, Dowse L, et al. Reducing vulnerability to harm in adults with cognitive disabilities in The Australian Criminal Justice System. J Policy Pract Intellect Disabil 2013;10:222-9.

23. Van Schrojenstein Lantman-De Valk HM, Metsemakers JF, Haveman MJ, et al. Health problems in people with intellectual disability in general practice: a comparative study. Fam Pract 2000;17:405-7.

24. Howlett $\mathrm{S}$, Florio $\mathrm{T}, \mathrm{Xu} \mathrm{H}$, et al. Ambulatory mental health data demonstrates the high needs of people with an intellectual disability: results from the New South Wales intellectual disability and mental health data linkage project. Aust $N Z J$ Psychiatry 2015;49:137-44.

25. Council of Australian Governments. National Disability Strategy 2010-2020. Canberra: Commonwealth of Australia, 2011.

26. National Centre for Classification in Health. The international statistical classification of diseases and related health problems, 10th revision, Australian modification (ICD-10-AM). Sydney: National Centre for Classification in Health, Faculty of Health Sciences, University of Sydney, 1998.

27. The Centre for Health Record Linkage. http://www.cherel.org.au/ data-dictionaries\#section2 (cited 22 April 2016).

28. The Centre for Health Record Linkage (CHeReL). How record linkage works. 2016. (15 December 2016) http://www.cherel.org.au/ how-record-linkage-works 